\title{
Quantization dimension for Gibbs-like measures on cookie-cutter sets
}

\author{
Mrinal Kanti Roychowdhury
}

\begin{abstract}
In this paper using the Banach limit we have determined a Gibbs-like measure $\mu_{h}$ supported by a cookie-cutter set $E$ which is generated by a single cookie-cutter mapping $f$. For such a measure $\mu_{h}$ and $r \in(0,+\infty)$ we have shown that there exists a unique $\kappa_{r} \in(0,+\infty)$ such that $\kappa_{r}$ is the quantization dimension function of the probability measure $\mu_{h}$, and we established its functional relationship with the temperature function of the thermodynamic formalism. The temperature function is commonly used to perform the multifractal analysis, in our context of the measure $\mu_{h}$. In addition, we have proved that the $\kappa_{r}$-dimensional lower quantization coefficient of order $r$ of the probability measure is positive.
\end{abstract}

\section{Introduction}

Quantization dimension is one of the most important objects in the quantization problem, which has a deep background in information theory and engineering technology (see $[\mathrm{BW}],[\mathrm{GG}],[\mathrm{GN}],[\mathrm{Z}]$ ). It characterizes in a natural way the asymptotic property of the error when approximating a given probability measure by a discrete probability measure of finite support in the sense of $L_{r}$-metrics. Given a Borel probability measure $\mu$ on $\mathbb{R}^{d}$, a number $r \in(0,+\infty)$, and a natural number $n \in \mathbb{N}$, the $n$th quantization error of order $r$ of $\mu$ is defined by

$$
V_{n, r}(\mu):=\inf \left\{\int d(x, \alpha)^{r} d \mu(x): \alpha \subset \mathbb{R}^{d}, \operatorname{card}(\alpha) \leq n\right\},
$$

where $d(x, \alpha)$ denotes the distance from the point $x$ to the set $\alpha$ with respect to a given norm $\|\cdot\|$ on $\mathbb{R}^{d}$. Note that if $\int\|x\|^{r} d \mu(x)<\infty$, then there is some set $\alpha$ for which the infimum is achieved (see [GL1]). The set $\alpha$ for which the infimum is achieved is called an optimal set of n-means or an n-optimal set of order $r$ for $0<r<+\infty$. The upper and lower quantization dimensions of order $r$ of $\mu$ are defined to be

$$
\bar{D}_{r}(\mu):=\limsup _{n \rightarrow \infty} \frac{r \log n}{-\log V_{n, r}(\mu)} ; \quad \underline{D}_{r}(\mu):=\liminf _{n \rightarrow \infty} \frac{r \log n}{-\log V_{n, r}(\mu)} .
$$

If $\bar{D}_{r}(\mu)$ and $\underline{D}_{r}(\mu)$ coincide, we call the common value the quantization dimension of order $r$ of the probability measure $\mu$, and it is denoted by $D_{r}:=D_{r}(\mu)$.

Kyoto Journal of Mathematics, Vol. 54, No. 2 (2014), 239-257

DOI $10.1215 / 21562261-2642377$, (C) 2014 by Kyoto University

Received August 22, 2012. Revised January 17, 2013. Accepted January 21, 2013.

2010 Mathematics Subject Classification: Primary 60Exx; Secondary 28A80, 94A34. 
For $s>0$, we define the $s$-dimensional upper and lower quantization coefficients of order $r$ of $\mu$ by $\limsup _{n} n V_{n, r}^{s / r}(\mu)$ and $\liminf _{n} n V_{n, r}^{s / r}(\mu)$, respectively. One sees that the quantization dimension is actually a function $r \mapsto D_{r}$ which measures the asymptotic rate at which $V_{n, r}$ goes to zero. If $D_{r}$ exists, then one can write

$$
\log V_{n, r} \sim \log \left[\left(\frac{1}{n}\right)^{r / D_{r}}\right] .
$$

For probabilities with nonvanishing absolutely continuous part the numbers $D_{r}$ are all equal to the dimension $d$ of the underlying space, but for singular probabilities the family $\left(D_{r}\right)_{r>0}$ gives an interesting description of their geometric (multifractal) structures.

Let $S_{1}, S_{2}, \ldots, S_{N}$ be contractive similitudes from $\mathbb{R}^{d}$ into itself, where $N \geq 2$ is a positive integer. Let $s_{i}$ be the contraction ratio of $S_{i}$ for all $1 \leq i \leq N$. Then for a given probability vector $\left(p_{1}, p_{2}, \ldots, p_{N}\right)$ there exists a unique Borel probability measure $\mu$ (see $[\mathrm{H}]$ ) satisfying the condition

$$
\mu=\sum_{j=1}^{N} p_{j} \mu \circ S_{j}^{-1} .
$$

Let the iterated function system $\left\{S_{1}, S_{2}, \ldots, S_{N}\right\}$ satisfy the open set condition (OSC): there exists a bounded nonempty open set $U \subset \mathbb{R}^{d}$ such that $\bigcup_{j=1}^{N} S_{j}(U) \subset$ $U$ and $S_{i}(U) \cap S_{j}(U)=\emptyset$ for $1 \leq i \neq j \leq N$. The iterated function system satisfies the strong OSC if $U$ can be chosen such that $U \cap J \neq \emptyset$, where $J$ is the limit set of the iterated function system. Under the OSC, Graf and Luschgy showed that the quantization dimension function $D_{r}:=D_{r}(\mu)$ of the probability measure $\mu$ exists and satisfies the following relation (see [GL1], [GL3]):

$$
\sum_{j=1}^{N}\left(p_{j} s_{j}^{r}\right)^{D_{r} /\left(r+D_{r}\right)}=1 .
$$

In fact, they proved a stronger result, namely, that the quantization dimension $D_{r}$ also satisfies (see [GL2])

$$
0<\liminf _{n} n V_{n, r}^{D_{r} / r}(\mu) \leq \underset{n}{\lim \sup } n V_{n, r}^{D_{r} / r}(\mu)<+\infty .
$$

Under the OSC, Lindsay and Mauldin [LM] determined the quantization dimension for an $F$-conformal measure $m$ associated with a conformal iterated function system determined by finitely many conformal mappings. They established a relationship between the quantization dimension and the temperature function of the thermodynamic formalism arising in multifractal analysis. Later, in [R1] the author studied the quantization dimension of Moran measures on the Moran sets of which potential functions are defined in terms of the similarity ratios and probability vectors. Then the quantization problem was solved in [R2] for the image measures of Bowen's Gibbs measures supported by the one-sided Bernoulli shifts under the coding maps on the Moran sets. The mixed quantization dimension and its relationship with the temperature function were studied by Wang and Dai [WD]. But from the work in [LM], [R1], [R2], and [WD] it was 
not known whether the $D_{r}$-dimensional lower quantization coefficient is positive, that is, whether $\liminf _{n} n V_{n, r}^{D_{r} / r}(\mu)>0$, where $D_{r}:=D_{r}(\mu)$ is the quantization dimension of the probability measure $\mu$.

In this paper, using the Banach limit we have defined a Gibbs-like measure $\mu_{h}$ supported by a cookie-cutter set $E$, where $E$ is the limit set generated by a cookie-cutter mapping $f$ and $h:=\operatorname{dim}_{\mathrm{H}}(E)$ is the Hausdorff dimension of the set $E$ (see [F2]). For this measure $\mu_{h}$ we have shown that for each $r \in(0,+\infty)$ there exists a unique $\kappa_{r} \in(0,+\infty)$ such that

$$
\lim _{k \rightarrow \infty} \frac{1}{k} \log \sum_{\sigma \in \Omega_{k}}\left(\mu_{h}\left(J_{\sigma}\right)\left\|\varphi_{\sigma}^{\prime}\right\|^{r}\right)^{\kappa_{r} /\left(r+\kappa_{r}\right)}=0,
$$

and the above $\kappa_{r}$ is the quantization dimension $D_{r}:=D_{r}\left(\mu_{h}\right)$ of order $r$ of the probability measure $\mu_{h}$. It is known that the singularity exponent $\beta(q)$ (also known as the temperature function) satisfies the usual equation

$$
\lim _{k \rightarrow \infty} \frac{1}{k} \log \sum_{\sigma \in \Omega_{k}}\left(\mu_{h}\left(J_{\sigma}\right)\right)^{q}\left\|\varphi_{\sigma}^{\prime}\right\|^{\beta(q)}=0
$$

and that the spectrum $f(\alpha)$ is the Legendre transform of $\beta(q)$. Comparing (2) and (3), we see that if $q_{r}=D_{r} /\left(r+D_{r}\right)$, then $\beta\left(q_{r}\right)=r q_{r}$; that is, the quantization dimension function of order $r$ of the probability measure $\mu_{h}$ has a relationship with the temperature function of the thermodynamic formalism arising in multifractal analysis. (For thermodynamic formalism, multifractal analysis, and the Legendre transform one could see [F2].) The significant difference between the work in this paper and the work in [LM], [R1], [R2], and [WD] is that, in addition to determining the quantization dimension function $\kappa_{r}$ and its relationship with the temperature function of a probability measure, by Proposition 3.10 in this paper, we have proved a stronger result, namely, that the $\kappa_{r}$-dimensional lower quantization coefficient $\liminf _{n} n V_{n, r}^{\kappa_{r} / r}\left(\mu_{h}\right)$ of order $r$ of the probability measure $\mu_{h}$ is positive. The quantization problem for a general probability measure is still open.

\section{Basic definitions, lemmas, and propositions}

In this paper, $\mathbb{R}^{d}$ denotes the $d$-dimensional Euclidean space equipped with a metric $d$ compatible with the Euclidean topology. Let us write

$$
\begin{aligned}
& V_{n, r}(\mu):=\inf \left\{\int d(x, \alpha)^{r} d \mu(x): \alpha \subset \mathbb{R}^{d}, \operatorname{card}(\alpha) \leq n\right\}, \\
& u_{n, r}(\mu):=\inf \left\{\int d\left(x, \alpha \cup U^{c}\right)^{r} d \mu(x): \alpha \subset \mathbb{R}^{d}, \operatorname{card}(\alpha) \leq n\right\},
\end{aligned}
$$

where $U$ is a set which comes from the OSC and $U^{c}$ denotes the complement of $U$. We see that

$$
u_{n, r}^{1 / r} \leq V_{n, r}^{1 / r}:=e_{n, r}
$$


We call sets $\alpha_{n} \subset \mathbb{R}^{d}$, for which the above infimums are achieved, $n$-optimal sets for $e_{n, r}, V_{n, r}$, or $u_{n, r}$, respectively. As stated above, Graf and Luschgy have shown that $n$-optimal sets exist when $\int\|x\|^{r} d \mu(x)<\infty$.

\subsection{Cookie-cutter set}

A mapping $f$ is called a cookie cutter if there exists a finite collection of disjoint closed intervals $J_{1}, J_{2}, \ldots, J_{N} \subset J=[0,1]$ such that

(C1) $f$ is defined in a neighborhood of each $J_{j}, 1 \leq j \leq N$, the restriction of $f$ to each initial interval $J_{j}$ maps $J_{j}$ bijectively onto $J$, and the corresponding branch inverse is denoted by $\varphi_{j}:=\left(\left.f\right|_{J_{j}}\right)^{-1}: J \rightarrow J_{j}$;

(C2) $f$ is differentiable with Hölder continuous derivative $f^{\prime}$, that is, there exist constants $c>0$ and $\gamma \in(0,1]$ such that for $x, y \in J_{j}, 1 \leq j \leq N$,

$$
\left|f^{\prime}(x)-f^{\prime}(y)\right| \leq c|x-y|^{\gamma}
$$

(C3) $f$ is boundedly expanding in the sense that there exist constants $b$ and $B$ such that

$$
1<b:=\inf _{x}\left\{\left|f^{\prime}(x)\right|\right\} \leq \sup _{x}\left\{\left|f^{\prime}(x)\right|\right\}:=B<+\infty .
$$

We call $\left[\bigcup_{j=1}^{N} J_{j} ; c, \gamma, b, B\right]$ the defining data of the cookie-cutter mapping $f$. Let $\Omega_{0}$ be the empty set. For $n \geq 1$, define

$$
\Omega_{n}=\{1,2, \ldots, N\}^{n}, \quad \Omega_{\infty}=\{1,2, \ldots, N\}^{\mathbb{N}}, \quad \text { and } \quad \Omega=\bigcup_{k=0}^{\infty} \Omega_{k} .
$$

Elements of $\Omega$ are called words. For any $\sigma \in \Omega$ if $\sigma=\left(\sigma_{1}, \sigma_{2}, \ldots, \sigma_{n}\right) \in \Omega_{n}$, we write $\sigma^{-}=\left(\sigma_{1}, \sigma_{2}, \ldots, \sigma_{n-1}\right)$ to denote the word obtained by deleting the last letter of $\sigma,|\sigma|=n$ to denote the length of $\sigma$, and $\left.\sigma\right|_{k}:=\left(\sigma_{1}, \sigma_{2}, \ldots, \sigma_{k}\right), k \leq n$, to denote the truncation of $\sigma$ to the length $k$. For any two words $\sigma=\left(\sigma_{1}, \sigma_{2}, \ldots, \sigma_{k}\right)$ and $\tau=\left(\tau_{1}, \tau_{2}, \ldots, \tau_{m}\right)$, we write $\sigma \tau=\sigma * \tau=\left(\sigma_{1}, \ldots, \sigma_{k}, \tau_{1}, \ldots, \tau_{m}\right)$ to denote the juxtaposition of $\sigma, \tau \in \Omega$. A word of length zero is called the empty word and is denoted by $\emptyset$. For $\sigma \in \Omega$ and $\tau \in \Omega \cup \Omega_{\infty}$ we say that $\tau$ is an extension of $\sigma$, written as $\sigma \prec \tau$, if $\left.\tau\right|_{|\sigma|}=\sigma$. For $\sigma \in \Omega_{k}$, the cylinder set $C(\sigma)$ is defined as $C(\sigma)=\left\{\tau \in \Omega_{\infty}:\left.\tau\right|_{k}=\sigma\right\}$. For $\sigma=\left(\sigma_{1}, \sigma_{2}, \ldots, \sigma_{n}\right) \in \Omega_{n}$, let us write $\varphi_{\sigma}=$ $\varphi_{\sigma_{1}} \circ \cdots \circ \varphi_{\sigma_{n}}$, and we define the rank- $n$ basic interval corresponding to $\sigma$ by

$$
J_{\sigma}=J_{\left(\sigma_{1}, \sigma_{2}, \ldots, \sigma_{n}\right)}=\varphi_{\sigma}(J),
$$

where $1 \leq \sigma_{k} \leq N, 1 \leq k \leq n$. If $\sigma=\emptyset$, then we identify $\varphi_{\emptyset}$ to be the identity mapping on $J$ and write $J_{\emptyset}=J$. By $\left|J_{\sigma}\right|$ we mean the diameter of the set $J_{\sigma}$ for all $\sigma \in \Omega$. It is easy to see that the set of basic intervals $\left\{J_{\sigma}: \sigma \in \Omega\right\}$ has the following net properties:

(i) $J_{\sigma * j} \subset J_{\sigma}$ for each $\sigma \in \Omega_{n}$ and $1 \leq j \leq N$ for all $n \geq 1$;

(ii) $J_{\sigma} \cap J_{\tau}=\emptyset$ if $\sigma, \tau \in \Omega_{n}$ for all $n \geq 1$ and $\sigma \neq \tau$. 
Since $\varphi_{j}$ is a branch inverse of $f$, where $1 \leq j \leq N$, for all $x \in J$, we have $f\left(\varphi_{j}(x)\right)=x$, and so $\left|f^{\prime}\left(\varphi_{j}(x)\right)\right| \cdot\left|\varphi_{j}^{\prime}(x)\right|=1$, which yields

$$
B^{-1} \leq\left|\varphi_{j}^{\prime}(x)\right| \leq b^{-1} \text {. }
$$

Choose $x, y$ to be the endpoints of $J$. Then $\varphi_{\sigma}(x), \varphi_{\sigma}(y)$ are the endpoints of $J_{\sigma}$ for each $\sigma \in \Omega$, and so by the mean value theorem, we have that

$$
\left|J_{\sigma}\right|=\left|\varphi_{\sigma}(x)-\varphi_{\sigma}(y)\right|=\left|\varphi_{\sigma}^{\prime}(w)\right||x-y|=\left|\varphi_{\sigma}^{\prime}(w)\right|
$$

for some $w \in J_{\sigma}$. Thus, $B^{-n} \leq\left|J_{\sigma}\right| \leq b^{-n}$ for any $\sigma \in \Omega_{n}$, and thus the diameter $\left|J_{\sigma}\right| \rightarrow 0$ as $|\sigma| \rightarrow \infty$. Since given $\sigma=\left(\sigma_{i}\right)_{i=1}^{\infty} \in \Omega_{\infty}$ the diameters of the compact sets $J_{\left.\sigma\right|_{k}}, k \geq 1$, converge to zero and since they form a descending family, the set

$$
\bigcap_{k=0}^{\infty} J_{\left.\sigma\right|_{k}}
$$

is a singleton, and therefore, if we denote its element by $\pi(\sigma)$, this defines the coding map $\pi: \Omega_{\infty} \rightarrow J$. The main object of our interest is the limit set

$$
E:=\pi\left(\Omega_{\infty}\right)=\bigcup_{\sigma \in \Omega_{\infty}} \bigcap_{k=0}^{\infty} J_{\left.\sigma\right|_{k}} .
$$

Moreover, $\pi(C(\sigma))=E \cap J_{\sigma}$ for $\sigma \in \Omega$. With the net properties it follows that $E$ is a perfect, nowhere dense, and totally disconnected subset of $J$. The set $E$ is called the cookie-cutter set.

Let $\ell^{\infty}$ be the set of all bounded sequences $x=\left(x_{n}\right)_{n \in \mathbb{N}}$ of real or complex numbers which form a vector space with respect to pointwise addition and multiplication by a scalar. It is equipped with the norm $\|x\|=\sup _{n}\left|x_{n}\right|$. The normed space $\ell^{\infty}$ is complete with respect to the metric $\|x-y\|$, and so it forms a Banach space. By the Hahn-Banach theorem (see [Y, pp. 102-104]), there exists a linear functional $L: \ell^{\infty} \rightarrow \mathbb{R}$ for which

(i) $L$ is linear;

(ii) $L\left(\left(x_{n}\right)_{n \in \mathbb{N}}\right)=L\left(\left(x_{n+1}\right)_{n \in \mathbb{N}}\right)$;

(iii) $\liminf _{n \rightarrow \infty}\left(x_{n}\right) \leq L\left(\left(x_{n}\right)_{n \in \mathbb{N}}\right) \leq \lim \sup _{n \rightarrow \infty}\left(x_{n}\right)$.

The functional $L$, defined above, is called a Banach limit. The use of the Banach limit is a rather standard tool in producing an invariant measure from a given measure.

Let us now prove the following lemma.

\section{LEMMA 2.2 (BOUNDED-VARIATION PRINCIPLE)}

There exists a constant $1<\xi<+\infty$ such that, for each $\sigma \in \Omega_{n}$ and $x, y \in J_{\sigma}$, we have

$$
\xi^{-1} \leq \frac{\left|\left(f^{n}\right)^{\prime}(x)\right|}{\left|\left(f^{n}\right)^{\prime}(y)\right|} \leq \xi
$$

where $f^{n}=f \circ f \circ \cdots \circ f$ represents the $n$-fold composition of $f$ with itself. 
Proof

Note that, for each $k \leq n$ and $\sigma=\left(\sigma_{1}, \sigma_{2}, \ldots, \sigma_{n}\right) \in \Omega_{n}, f^{k-1}$ maps $J_{\sigma}$ diffeomorphically to the set $\varphi_{\sigma_{k}} \circ \varphi_{\sigma_{k+1}} \circ \cdots \circ \varphi_{\sigma_{n}}(J)$, and so

$$
\begin{aligned}
\left|f^{k-1}(x)-f^{k-1}(y)\right| & \leq \operatorname{diam}\left(\varphi_{\sigma_{k}} \circ \varphi_{\sigma_{k+1}} \circ \cdots \circ \varphi_{\sigma_{n}}(J)\right) \\
& =\left|\varphi_{\sigma_{k}} \circ \varphi_{\sigma_{k+1}} \circ \cdots \circ \varphi_{\sigma_{n}}(J)\right| .
\end{aligned}
$$

By the mean value theorem,

$$
\begin{aligned}
& \left|\varphi_{\sigma_{k}} \circ \varphi_{\sigma_{k+1}} \circ \cdots \circ \varphi_{\sigma_{n}}(J)\right| \\
& \quad=\sup _{x, y \in J}\left|\varphi_{\sigma_{k}}\left(\varphi_{\sigma_{k+1}} \circ \cdots \circ \varphi_{\sigma_{n}}(x)\right)-\varphi_{\sigma_{k}}\left(\varphi_{\sigma_{k+1}} \circ \cdots \circ \varphi_{\sigma_{n}}(y)\right)\right| \\
& \quad \leq b^{-1}\left|\varphi_{\sigma_{k+1}} \circ \cdots \circ \varphi_{\sigma_{n}}(J)\right| .
\end{aligned}
$$

Thus, proceeding inductively, we have that

$$
\left|\varphi_{\sigma_{k}} \circ \varphi_{\sigma_{k+1}} \circ \cdots \circ \varphi_{\sigma_{n}}(J)\right| \leq b^{-(n-k+1)} .
$$

Then, the Hölder continuity of $f^{\prime}$ gives

$$
\left|f^{\prime}\left(f^{k-1}(x)\right)-f^{\prime}\left(f^{k-1}(y)\right)\right| \leq c\left|f^{k-1}(x)-f^{k-1}(y)\right|^{\gamma} \leq c b^{-(n-k+1) \gamma},
$$

and so by the mean value theorem and the assumption that $\left|f^{\prime}\right|>1$, we have

$$
\begin{aligned}
& |\log | f^{\prime}\left(f^{k-1}(x)\right)|-\log | f^{\prime}\left(f^{k-1}(y)\right)|| \\
& \quad \leq\left\|f^{\prime}\left(f^{k-1}(x)\right)|-| f^{\prime}\left(f^{k-1}(y)\right)\right\| \\
& \quad \leq c b^{-(n-k+1) \gamma} .
\end{aligned}
$$

Therefore, by the above inequality and the chain rule,

$$
\begin{aligned}
& |\log |\left(f^{n}\right)^{\prime}(x)|-\log |\left(f^{n}\right)^{\prime}(y)|| \\
& \quad=\left|\sum_{k=1}^{n} \log \right| f^{\prime}\left(f^{k-1}(x)\right)\left|-\sum_{k=1}^{n} \log \right| f^{\prime}\left(f^{k-1}(y)\right)|| \\
& \quad \leq \sum_{k=1}^{n}|\log | f^{\prime}\left(f^{k-1}(x)\right)|-\log | f^{\prime}\left(f^{k-1}(y)\right)|| \\
& \quad \leq \sum_{k=1}^{n} c b^{-(n-k+1) \gamma} \leq \frac{c b^{-\gamma}}{1-b^{-\gamma}} .
\end{aligned}
$$

Take $\xi=\exp \left\{c /\left(b^{\gamma}-1\right)\right\}$. Since $c /\left(b^{\gamma}-1\right)>0$, we have $1<\xi<+\infty$, and thus the lemma follows.

Let us now prove the following proposition.

PROPOSITION 2.3 (BOUNDED DISTORTION PRINCIPLE)

For any $n \geq 1, \sigma \in \Omega_{n}, x \in J_{\sigma}$, we have

$$
\xi^{-1} \leq\left|\left(f^{n}\right)^{\prime}(x)\right| \cdot\left|J_{\sigma}\right| \leq \xi .
$$


Moreover, for each $1 \leq j \leq N$, we get $\left|J_{\sigma * j}\right| \geq \xi^{-2} B^{-1}\left|J_{\sigma}\right|$, where $\xi$ is the constant of Lemma 2.2.

Proof

Note that, for $\sigma \in \Omega_{n}, f^{n}: J_{\sigma} \rightarrow J$ is a differentiable bijection. So by the mean value theorem, if $y, z \in J_{\sigma}$, there exists $w \in J_{\sigma}$ such that

$$
f^{n}(y)-f^{n}(z)=\left(f^{n}\right)^{\prime}(w)(y-z) .
$$

Choose $y, z$ to be the endpoints of $J_{\sigma}$. Then $f^{n}(y), f^{n}(z)$ are the endpoints of $J$, and so

$$
|J|=\left|\left(f^{n}\right)^{\prime}(w)\right| \cdot\left|J_{\sigma}\right| ; \quad \text { that is, } \quad\left|\left(f^{n}\right)^{\prime}(w)\right| \cdot\left|J_{\sigma}\right|=1 .
$$

Hence, using the bounded-variation principle, we have

$$
\xi^{-1} \leq\left|\left(f^{n}\right)^{\prime}(x)\right| \cdot\left|J_{\sigma}\right| \leq \xi
$$

for all $x \in J_{\sigma}$. Now let $1 \leq j \leq N$ and $x \in J_{\sigma * j}$. Then using (5), we have

$$
\begin{aligned}
\xi^{-1} & \leq\left|\left(f^{n+1}\right)^{\prime}(x)\right| \cdot\left|J_{\sigma * j}\right| \\
& =\mid\left(f^{\prime}\left(f^{n}(x)\right)|\cdot|\left(f^{n}\right)^{\prime}(x)|\cdot| J_{\sigma * j}|\leq B|\left(f^{n}\right)^{\prime}(x)|\cdot| J_{\sigma * j} \mid .\right.
\end{aligned}
$$

Since $J_{\sigma * j} \subseteq J_{\sigma}$, we have $x \in J_{\sigma}$. Hence, using (5) again, we have

$$
\left|J_{\sigma * j}\right| \geq \xi^{-2} B^{-1}\left|J_{\sigma}\right| \text {. }
$$

Thus, the proof of the proposition is yielded.

PROPOSITION 2.4

For any $n \geq 1$, let $\sigma \in \Omega_{n}$, and let $x, y \in J$. Let $\xi$ be the constant of Lemma 2.2. Then,

$$
\xi^{-1}\left|\varphi_{\sigma}^{\prime}(y)\right| \leq\left|\varphi_{\sigma}^{\prime}(x)\right| \leq \xi\left|\varphi_{\sigma}^{\prime}(y)\right|
$$

Proof

For $\sigma \in \Omega_{n}$ and $x \in J$, we know that $f^{n}\left(\varphi_{\sigma}(x)\right)=x$. Thus,

$$
\left|\left(f^{n}\right)^{\prime}\left(\varphi_{\sigma}(x)\right)\right| \cdot\left|\varphi_{\sigma}^{\prime}(x)\right|=1 .
$$

Again, for all $x \in J, \varphi_{\sigma}(x) \in J_{\sigma}$. Hence, Lemma 2.2 yields

$$
\xi^{-1}\left|\varphi_{\sigma}^{\prime}(y)\right| \leq\left|\varphi_{\sigma}^{\prime}(x)\right| \leq \xi\left|\varphi_{\sigma}^{\prime}(y)\right|
$$

and thus the proposition is obtained.

Let us now prove the following two lemmas.

\section{LEMMA 2.5}

Let $\sigma, \tau \in \Omega$. Then

$$
\xi^{-1}\left\|\varphi_{\sigma}^{\prime}\right\|\left\|\varphi_{\tau}^{\prime}\right\| \leq\left\|\varphi_{\sigma \tau}^{\prime}\right\| \leq\left\|\varphi_{\sigma}^{\prime}\right\|\left\|\varphi_{\tau}^{\prime}\right\|
$$

where $\xi$ is the constant of Lemma 2.2. 


\section{Proof}

For any $x \in J$, we have $\left|\varphi_{\sigma \tau}^{\prime}(x)\right|=\left|\varphi_{\sigma}^{\prime}\left(\varphi_{\tau}(x)\right)\right| \cdot\left|\varphi_{\tau}^{\prime}(x)\right|$. Thus, by Proposition 2.4, for any $y \in J$,

$$
\begin{aligned}
\xi^{-1}\left|\varphi_{\sigma}^{\prime}(y)\right| \cdot\left|\varphi_{\tau}^{\prime}(x)\right| & \leq\left|\varphi_{\sigma}^{\prime}\left(\varphi_{\tau}(x)\right)\right| \cdot\left|\varphi_{\tau}^{\prime}(x)\right| \\
& =\left|\varphi_{\sigma \tau}^{\prime}(x)\right| \leq\left\|\varphi_{\sigma}^{\prime}\right\| \cdot\left\|\varphi_{\tau}^{\prime}\right\|,
\end{aligned}
$$

and thus the lemma follows.

\section{LEMMA 2.6}

Let $\sigma \in \Omega$, and let $x \in J$. Then

$$
\xi^{-1}\left|J_{\sigma}\right| \leq\left|\varphi_{\sigma}^{\prime}(x)\right| \leq \xi\left|J_{\sigma}\right|,
$$

where $\xi$ is the constant of Lemma 2.2.

\section{Proof}

Let $x \in J$, and then $\varphi_{\sigma}(x) \in J_{\sigma}$ for $\sigma \in \Omega_{n}, n \geq 1$. We know that $f^{n}\left(\varphi_{\sigma}(x)\right)=x$, and so $\left|\left(f^{n}\right)^{\prime}\left(\varphi_{\sigma}(x)\right)\right| \cdot\left|\varphi_{\sigma}^{\prime}(x)\right|=1$. Now use Proposition 2.3 to obtain the lemma.

Let us now prove the following lemma.

\section{LEMMA 2.7}

Let $\sigma, \tau \in \Omega$. Then

$$
\xi^{-3}\left|J_{\sigma}\right|\left|J_{\tau}\right| \leq\left|J_{\sigma \tau}\right| \leq \xi^{3}\left|J_{\sigma}\right|\left|J_{\tau}\right|,
$$

where $\xi$ is the constant of Lemma 2.2.

Proof

For $\sigma, \tau \in \Omega$, we have $\left|\varphi_{\sigma \tau}^{\prime}(x)\right|=\left|\varphi_{\sigma}^{\prime}(y)\right|\left|\varphi_{\tau}^{\prime}(x)\right|$, where $y=\varphi_{\tau}(x)$ and $x \in J$. Again by Proposition 2.4, for any $x, y \in J$, we have

$$
\xi^{-1}\left|\varphi_{\sigma}^{\prime}(y)\right| \leq\left|\varphi_{\sigma}^{\prime}(x)\right| \leq \xi\left|\varphi_{\sigma}^{\prime}(y)\right| .
$$

Hence, Lemma 2.6 implies that

$$
\xi^{-3}\left|J_{\sigma}\right|\left|J_{\tau}\right| \leq \xi^{-1}\left|\varphi_{\sigma}^{\prime}(y)\right|\left|\varphi_{\tau}^{\prime}(x)\right|=\xi^{-1}\left|\varphi_{\sigma \tau}^{\prime}(x)\right| \leq\left|J_{\sigma \tau}\right| \leq \xi\left|\varphi_{\sigma \tau}^{\prime}(x)\right| \leq \xi^{3}\left|J_{\sigma}\right|\left|J_{\tau}\right|,
$$

and thus the lemma is obtained.

By Lemma 2.5 and the standard theory of subadditive sequences, the function $Q(t)$ given by

$$
Q(t)=\lim _{k \rightarrow \infty} \frac{1}{k} \log \sum_{\sigma \in \Omega_{k}}\left\|\varphi_{\sigma}^{\prime}\right\|^{t},
$$

for any real $t$, exists. It is easy to see that the function $Q(t)$ is strictly decreasing, convex and hence continuous in $t$. 


\section{LEMMA 2.8}

There exists a unique $h \in(0,+\infty)$ such that $Q(h)=0$.

Proof

Since the function $Q(t)$ is strictly decreasing and continuous on $\mathbb{R}$, there exists a unique $h \in \mathbb{R}$ such that $Q(h)=0$. Note that

$$
Q(0)=\lim _{k \rightarrow \infty} \frac{1}{k} \log \sum_{\sigma \in \Omega_{k}} 1 \geq \lim _{k \rightarrow \infty} \frac{1}{k} \log N^{k}=\log N \geq \log 2>0 .
$$

In order to conclude the proof, it therefore suffices to show that $\lim _{t \rightarrow+\infty} Q(t)=$ $-\infty$. For $t>0$,

$$
\begin{aligned}
Q(t) & =\lim _{k \rightarrow \infty} \frac{1}{k} \log \sum_{\sigma \in \Omega_{k}}\left\|\varphi_{\sigma}^{\prime}\right\|^{t} \leq \lim _{k \rightarrow \infty} \frac{1}{k} \log \sum_{\sigma \in \Omega_{k}} b^{-k t} \\
& =\lim _{k \rightarrow \infty} \frac{1}{k} \log N^{k}-t \log b=\log N-t \log b .
\end{aligned}
$$

Since $b>1$, it follows that $\lim _{t \rightarrow+\infty} Q(t)=-\infty$, and hence the lemma follows.

\section{NOTE 2.9}

Lemma 2.6 implies that $\xi^{-1}\left|J_{\sigma}\right| \leq \sup _{x \in J}\left|\varphi_{\sigma}^{\prime}(x)\right|=\left\|\varphi_{\sigma}^{\prime}\right\| \leq \xi\left|J_{\sigma}\right|$, and so the topological pressure $Q(t)$ can be written as follows:

$$
Q(t)=\lim _{k \rightarrow \infty} \frac{1}{k} \log \sum_{\sigma \in \Omega_{k}}\left|J_{\sigma}\right|^{t}
$$

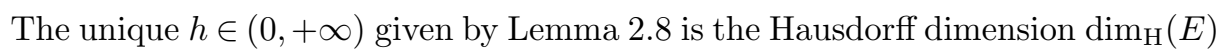
of the cookie-cutter set $E$ (see [F2]).

Let us now prove the following proposition, which plays a vital role in the paper.

PROPOSITION 2.10

Let $h \in(0,+\infty)$ be unique such that $Q(h)=0$, and let $s_{*}$ and $s^{*}$ be any two arbitrary real numbers with $0<s_{*}<h<s^{*}$. Then for all $n \geq 1$,

$$
\xi^{-3 s_{*}}<\sum_{\sigma \in \Omega_{n}}\left|J_{\sigma}\right|^{s_{*}} \quad \text { and } \quad \sum_{\sigma \in \Omega_{n}}\left|J_{\sigma}\right|^{s^{*}}<\xi^{3 s^{*}}
$$

where $\xi$ is the constant of Lemma 2.2.

Proof

Let $s_{*}<h$. As the pressure function $Q(t)$ is strictly decreasing, $Q\left(s_{*}\right)>Q(h)=0$. Then for any positive integer $n$, by Lemma 2.7 , we have

$$
0<Q\left(s_{*}\right)=\lim _{p \rightarrow \infty} \frac{1}{n p} \log \sum_{\omega \in \Omega_{n p}}\left|J_{\omega}\right|^{s_{*}} \leq \lim _{p \rightarrow \infty} \frac{1}{n p} \log \xi^{3(p-1) s_{*}}\left(\sum_{\sigma \in \Omega_{n}}\left|J_{\sigma}\right|^{s_{*}}\right)^{p}
$$


which implies that

$$
0<\frac{1}{n} \log \left(\xi^{3 s_{*}} \sum_{\sigma \in \Omega_{n}}\left|J_{\sigma}\right|^{s_{*}}\right), \quad \text { and so } \quad \sum_{\sigma \in \Omega_{n}}\left|J_{\sigma}\right|^{s_{*}}>\xi^{-3 s_{*}} .
$$

Now if $h<s^{*}$, then $Q\left(s^{*}\right)<0$ as $Q(t)$ is strictly decreasing. Then for any positive integer $n$, by Lemma 2.7 , we have

$$
0>Q\left(s^{*}\right)=\lim _{p \rightarrow \infty} \frac{1}{n p} \log \sum_{\omega \in \Omega_{n p}}\left|J_{\omega}\right|^{s^{*}} \geq \lim _{p \rightarrow \infty} \frac{1}{n p} \log \xi^{-3(p-1) s^{*}}\left(\sum_{\sigma \in \Omega_{n}}\left|J_{\sigma}\right|^{s^{*}}\right)^{p},
$$

which implies that

$$
0>\frac{1}{n} \log \left(\xi^{-3 s^{*}} \sum_{\sigma \in \Omega_{n}}\left|J_{\sigma}\right|^{s^{*}}\right), \quad \text { and so } \quad \sum_{\sigma \in \Omega_{n}}\left|J_{\sigma}\right|^{s^{*}}<\xi^{3 s^{*}} .
$$

Thus, the proposition is obtained.

\section{COROLLARY 2.11}

Since $s_{*}$ and $s^{*}$ are any two arbitrary real numbers with $0<s_{*}<h<s^{*}$, from the above proposition it follows that, for all $n \geq 1$,

$$
\xi^{-3 h} \leq \sum_{\sigma \in \Omega_{n}}\left|J_{\sigma}\right|^{h} \leq \xi^{3 h} .
$$

Let us now prove the following proposition.

\section{PROPOSITION 2.12 (GIBBS-LIKE MEASURE)}

Let $h \in(0,+\infty)$ be such that $Q(h)=0$. Then there exist a constant $\eta>1$ and a probability measure $\mu_{h}$ supported by $E$ such that, for any $\sigma \in \Omega$,

$$
\eta^{-1}\left|J_{\sigma}\right|^{h} \leq \mu_{h}\left(J_{\sigma}\right) \leq \eta\left|J_{\sigma}\right|^{h} \text {. }
$$

Proof

For $\sigma \in \Omega, n \geq 1$, define

$$
\nu_{n}(C(\sigma))=\frac{\sum_{\tau \in \Omega_{n}}\left(\operatorname{diam} J_{\sigma \tau}\right)^{h}}{\sum_{\tau \in \Omega_{|\sigma|+n}}\left(\operatorname{diam} J_{\tau}\right)^{h}} .
$$

Then using Lemma 2.7 and Corollary 2.11, we have

$$
\nu_{n}(C(\sigma)) \leq \frac{\xi^{3 h}\left(\operatorname{diam} J_{\sigma}\right)^{h} \sum_{\tau \in \Omega_{n}}\left(\operatorname{diam} J_{\tau}\right)^{h}}{\sum_{\tau \in \Omega_{|\sigma|+n}}\left(\operatorname{diam} J_{\tau}\right)^{h}} \leq \xi^{9 h}\left(\operatorname{diam} J_{\sigma}\right)^{h},
$$

and similarly, $\nu_{n}(C(\sigma)) \geq \xi^{-9 h}\left(\operatorname{diam} J_{\sigma}\right)^{h}$. Thus, for a given $\sigma \in \Omega,\left\{\nu_{n}(C(\sigma))\right\}_{n=1}^{\infty}$ is a bounded sequence of real numbers, and so the Banach limit, denoted by Lim, is defined. For $\sigma \in \Omega$, let

$$
\nu(C(\sigma))=\operatorname{Lim}_{n \rightarrow \infty} \nu_{n}(C(\sigma)) .
$$


Then

$$
\begin{aligned}
\sum_{j=1}^{N} \nu(C(\sigma j)) & =\operatorname{Lim}_{n \rightarrow \infty} \sum_{j=1}^{N} \frac{\sum_{\tau \in \Omega_{n}}\left(\operatorname{diam} J_{\sigma j \tau}\right)^{h}}{\sum_{\tau \in \Omega_{|\sigma|+1+n}}\left(\operatorname{diam} J_{\tau}\right)^{h}} \\
& =\operatorname{Lim}_{n \rightarrow \infty} \frac{\sum_{\tau \in \Omega_{n+1}}\left(\operatorname{diam} J_{\sigma \tau}\right)^{h}}{\sum_{\tau \in \Omega_{|\sigma|+n+1}}\left(\operatorname{diam} J_{\tau}\right)^{h}}
\end{aligned}
$$

and so

$$
\sum_{j=1}^{N} \nu(C(\sigma j))=\operatorname{Lim}_{n \rightarrow \infty} \nu_{n+1}(C(\sigma))=\operatorname{Lim}_{n \rightarrow \infty} \nu_{n}(C(\sigma))=\nu(C(\sigma)) .
$$

Thus, by Kolmogorov's extension theorem, $\nu$ can be extended to a unique Borel probability measure $\gamma$ on $\Omega_{\infty}$. Let $\mu_{h}$ be the image measure of $\gamma$ under the coding map $\pi$; that is, $\mu_{h}=\gamma \circ \pi^{-1}$. Then $\mu_{h}$ is a unique Borel probability measure supported by $E$. Moreover, for any $\sigma \in \Omega$,

$$
\begin{aligned}
\mu_{h}\left(J_{\sigma}\right) & =\gamma(C(\sigma))=\operatorname{Lim}_{n \rightarrow \infty} \nu_{n}(C(\sigma)) \\
& \leq \operatorname{Lim}_{n \rightarrow \infty} \xi^{9 h}\left(\operatorname{diam} J_{\sigma}\right)^{h}=\xi^{9 h}\left(\operatorname{diam} J_{\sigma}\right)^{h},
\end{aligned}
$$

and similarly,

$$
\mu_{h}\left(J_{\sigma}\right) \geq \xi^{-9 h}\left(\operatorname{diam} J_{\sigma}\right)^{h} .
$$

Write $\eta=\xi^{9 h}$, and then $\eta>1$, and thus the proof of the proposition is complete.

For the above measure $\mu_{h}$, known as a Gibbs-like measure, we will determine the quantization dimension function and its functional relationship with the temperature function of the thermodynamic formalism.

Let us now prove the following lemma.

\section{LEMMA 2.13}

Let $\mu_{h}$ be the Gibbs-like measure as defined in Proposition 2.12. Then there exists a constant $L>1$ such that, for $\sigma, \tau \in \Omega$,

$$
L^{-1} \mu_{h}\left(J_{\sigma}\right) \mu_{h}\left(J_{\tau}\right) \leq \mu_{h}\left(J_{\sigma \tau}\right) \leq L \mu_{h}\left(J_{\sigma}\right) \mu_{h}\left(J_{\tau}\right) .
$$

Proof

Let $\sigma, \tau \in \Omega$. Then by Lemma 2.7 and Proposition 2.12, we have

$$
\mu_{h}\left(J_{\sigma \tau}\right) \leq \eta\left|J_{\sigma \tau}\right|^{h} \leq \eta \xi^{3 h}\left|J_{\sigma}\right|^{h}\left|J_{\tau}\right|^{h} \leq \eta^{3} \xi^{3 h} \mu_{h}\left(J_{\sigma}\right) \mu_{h}\left(J_{\eta}\right),
$$

and similarly, $\mu_{h}\left(J_{\sigma \tau}\right) \geq \eta^{-3} \xi^{-3 h} \mu_{h}\left(J_{\sigma}\right) \mu_{h}\left(J_{\eta}\right)$. Take $L=\eta^{3} \xi^{3 h}$. As $h>0, \xi>1$, and $\eta>1$, it follows that $L>1$, and thus

$$
L^{-1} \mu_{h}\left(J_{\sigma}\right) \mu_{h}\left(J_{\eta}\right) \leq \mu_{h}\left(J_{\sigma \tau}\right) \leq L \mu_{h}\left(J_{\sigma}\right) \mu_{h}\left(J_{\eta}\right),
$$

which is the lemma. 


\subsection{Topological pressure}

For $q, t \in \mathbb{R}$ and $n \geq 1$, let us write

$$
Z_{n}(q, t)=\sum_{\sigma \in \Omega_{n}}\left(\mu_{h}\left(J_{\sigma}\right)\right)^{q}\left\|\varphi_{\sigma}^{\prime}\right\|^{t} .
$$

Then for $n, p \geq 1$,

$$
Z_{n+p}(q, t)=\sum_{\sigma \in \Omega_{n}} \sum_{\tau \in \Omega_{p}}\left(\mu_{h}\left(J_{\sigma \tau}\right)\right)^{q}\left\|\varphi_{\sigma \tau}^{\prime}\right\|^{t} .
$$

Let us first assume that $q \geq 0$. Then by Lemmas 2.5 and 2.13 , it follows that if $t \geq 0$, then

$$
Z_{n+p}(q, t) \leq L^{q} Z_{n}(q, t) Z_{p}(q, t)
$$

and if $t<0$, then

$$
Z_{n+p}(q, t) \leq L^{q} \xi^{-t} Z_{n}(q, t) Z_{p}(q, t) .
$$

Let us now assume that $q<0$. Using the same argument, we have that if $t \geq 0$, then

$$
Z_{n+p}(q, t) \leq L^{-q} Z_{n}(q, t) Z_{p}(q, t)
$$

and if $t<0$, then

$$
Z_{n+p}(q, t) \leq L^{-q} \xi^{-t} Z_{n}(q, t) Z_{p}(q, t)
$$

Hence, by the standard theory of subadditive sequences, $\lim _{k \rightarrow \infty}(1 / k) \log Z_{k}(q, t)$ exists (see [F2, Corollary 1.2]). Let us denote it by $P(q, t)$, that is,

$$
P(q, t)=\lim _{k \rightarrow \infty} \frac{1}{k} \log \sum_{\sigma \in \Omega_{k}}\left(\mu_{h}\left(J_{\sigma}\right)\right)^{q}\left\|\varphi_{\sigma}^{\prime}\right\|^{t} .
$$

The following proposition states the well-known properties of the function $P(q, t)$ (see $[\mathrm{F} 1],[\mathrm{P}])$.

\section{PROPOSITION 2.15}

(i) $\quad P(q, t): \mathbb{R} \times \mathbb{R} \rightarrow \mathbb{R}$ is continuous.

(ii) $P(q, t)$ is strictly decreasing in each variable separately.

(iii) For fixed $q$ we have $\lim _{t \rightarrow+\infty} P(q, t)=-\infty$ and $\lim _{t \rightarrow-\infty} P(q, t)=+\infty$.

(iv) $P(q, t)$ is convex: if $q_{1}, q_{2}, t_{1}, t_{2} \in \mathbb{R}, a_{1}, a_{2} \geq 0, a_{1}+a_{2}=1$, then

$$
P\left(a_{1} q_{1}+a_{2} q_{2}, a_{1} t_{1}+a_{2} t_{2}\right) \leq a_{1} P\left(q_{1}, t_{1}\right)+a_{2} P\left(q_{2}, t_{2}\right) .
$$

Now for fixed $q, P(q, t)$ is a continuous function of $t$. Its values range from $-\infty$ (when $t \rightarrow+\infty$ ) to $+\infty$ (when $t \rightarrow-\infty$ ). Therefore, by the intermediate value theorem there is a real number $\beta$ such that $P(q, \beta)=0$. The solution $\beta$ is unique, since $P(q, \cdot)$ is strictly decreasing. This defines $\beta$ implicitly as a function of $q$ : for each $q$ there is a unique $\beta=\beta(q)$ such that $P(q, \beta(q))=0$.

The following proposition gives the well-known properties of the function $\beta(q)$ (see $[\mathrm{F} 1],[\mathrm{P}])$. 
PROPOSITION 2.16

Let $\beta=\beta(q)$ be defined by $P(q, \beta(q))=0$. Then

(i) $\beta$ is a continuous function of the real variable $q$;

(ii) $\beta$ is strictly decreasing: if $q_{1}<q_{2}$, then $\beta\left(q_{1}\right)>\beta\left(q_{2}\right)$;

(iii) $\lim _{q \rightarrow-\infty} \beta(q)=+\infty$ and $\lim _{q \rightarrow+\infty} \beta(q)=-\infty$;

(iv) $\beta$ is convex: if $q_{1}, q_{2}, a_{1}, a_{2} \in \mathbb{R}$ with $a_{1}, a_{2} \geq 0$ and $a_{1}+a_{2}=1$, then

$$
\beta\left(a_{1} q_{1}+a_{2} q_{2}\right) \leq a_{1} \beta\left(q_{1}\right)+a_{2} \beta\left(q_{2}\right) .
$$

The function $\beta(q)$ is sometimes denoted by $T(q)$ and called the temperature function. A more general discussion of this function can be found in [HJK+], where our $\beta(q)$ function corresponds to $-\tau(q)$ in their notation.

\section{REMARK 2.17}

If $q=0$, then $P(0, \beta(0))=0$, which implies that

$$
\lim _{k \rightarrow \infty} \frac{1}{k} \log \sum_{\sigma \in \Omega_{k}}\left\|\varphi_{\sigma}^{\prime}\right\|^{\beta(0)}=0 ;
$$

that is, $\beta(0)$ gives the Hausdorff dimension $\operatorname{dim}_{H}(E)$ of the cookie-cutter set $E$ (see [F2]). Again,

$$
P(1,0)=\lim _{k \rightarrow \infty} \frac{1}{k} \log \sum_{\sigma \in \Omega_{k}} \mu_{h}\left(J_{\sigma}\right)=\lim _{k \rightarrow \infty} \frac{1}{k} \log 1=0,
$$

and hence $\beta(1)=0$ (see Figure 1$)$.

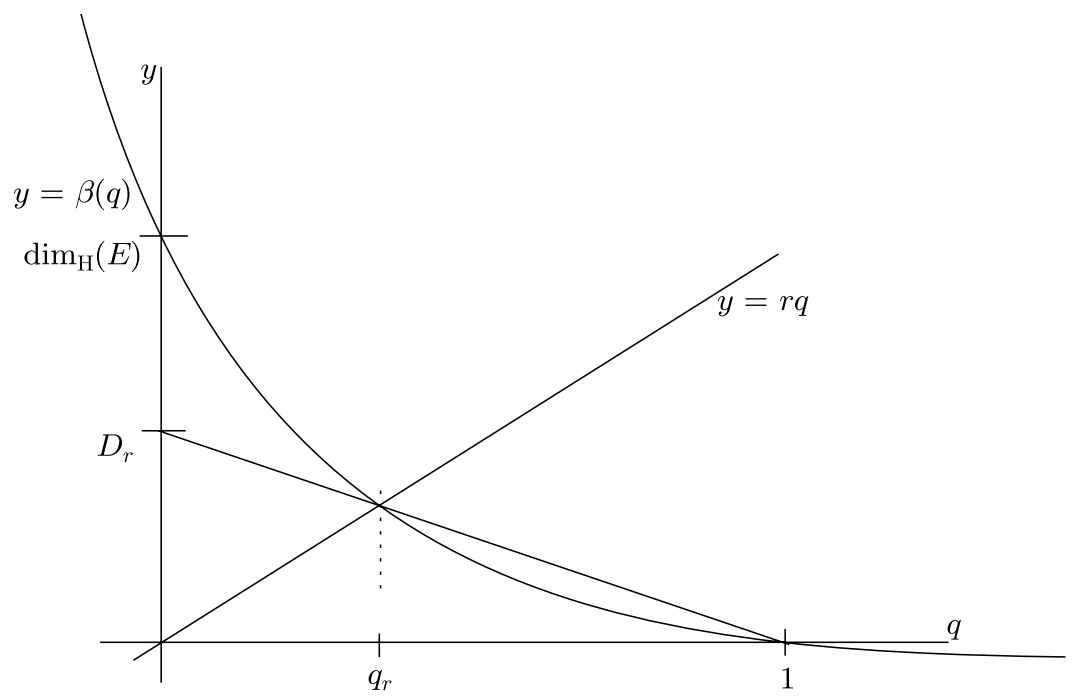

Figure 1. To determine $D_{r}$ first find the point of intersection of $y=\beta(q)$ and the line $y=r q$. Then $D_{r}$ is the $y$-intercept of the line through this point and the point $(1,0)$. 


\section{Main result}

The relationship between the quantization dimension function and the temperature function $\beta(q)$ for the Gibbs-like measure $\mu_{h}$, where the temperature function is the Legendre transform of the $f(\alpha)$ curve (for the definitions of $f(\alpha)$ and the Legendre transform see [F2]), is given by the following theorem, which constitutes the main result of the paper. For its graphical description, see Figure 1.

\section{THEOREM 3.1}

Let $\mu_{h}$ be the Gibbs-like measure supported by the cookie-cutter set E. Then, for each $r \in(0,+\infty)$, there exists a unique $\kappa_{r} \in(0,+\infty)$ such that

$$
\kappa_{r}=\frac{\beta\left(q_{r}\right)}{1-q_{r}},
$$

where we recall $\beta$ is the temperature function, that is, $\beta\left(q_{r}\right)=r q_{r}$, and $\kappa_{r}$ is the quantization dimension of order $r$ of the probability measure $\mu_{h}$. Moreover, the $\kappa_{r}$-dimensional lower quantization coefficient is positive, that is, $\liminf _{n} n V_{n, r}^{\kappa_{r} / r}\left(\mu_{h}\right)>0$.

To prove the theorem we need several lemmas and propositions. Let us first state the following lemma, which has a proof similar to that of [R2, Lemma 3.2].

\section{LEMMA 3.2}

Let $0<r<+\infty$ be fixed. Then there exists exactly one number $\kappa_{r} \in(0,+\infty)$ such that

$$
\lim _{k \rightarrow \infty} \frac{1}{k} \log \sum_{\sigma \in \Omega_{k}}\left(\mu_{h}\left(J_{\sigma}\right)\left\|\varphi_{\sigma}^{\prime}\right\|^{r}\right)^{\kappa_{r} /\left(r+\kappa_{r}\right)}=0
$$

Let us now prove the following lemma.

LEMMA 3.3

Let $0<r<+\infty$ and $\kappa_{r}$ be as in Lemma 3.2. Then for $n \geq 1$, we have

$$
\left(L \xi^{r}\right)^{-\kappa_{r} /\left(r+\kappa_{r}\right)} \leq \sum_{\sigma \in \Omega_{n}}\left(\mu_{h}\left(J_{\sigma}\right)\left\|\varphi_{\sigma}^{\prime}\right\|^{r}\right)^{\kappa_{r} /\left(r+\kappa_{r}\right)} \leq\left(L \xi^{r}\right)^{\kappa_{r} /\left(r+\kappa_{r}\right)} .
$$

Proof

For $\sigma \in \Omega$ let us write $s_{\sigma}=\mu_{h}\left(J_{\sigma}\right)\left\|\varphi_{\sigma}^{\prime}\right\|^{r}$. Then for $\sigma \in \Omega_{n}$ and $\tau \in \Omega_{p}$ with $n, p \geq 1$, by Lemmas 2.5 and 2.13, we have $L^{-1} \xi^{-r} s_{\sigma} s_{\tau} \leq s_{\sigma \tau} \leq L s_{\sigma} s_{\tau} \leq L \xi^{r} s_{\sigma} s_{\tau}$. Since $r>0$ and $L, \xi>1$, it is true that $L^{-2} \xi^{-2 r} s_{\sigma} s_{\tau} \leq s_{\sigma \tau} \leq L^{2} \xi^{2 r} s_{\sigma} s_{\tau}$. Then by the standard theory of subadditive sequences, $\lim _{n \rightarrow \infty} n^{-1} \log \sum_{\sigma \in \Omega_{n}} s_{\sigma}^{t}$ exists for any $t \in \mathbb{R}$. Then, proceeding as in [R2, Lemma 3.3], we obtain the lemma.

We call $\Gamma \subset \Omega$ a finite maximal antichain if $\Gamma$ is a finite set of words in $\Omega$ such that every sequence in $\Omega_{\infty}$ is an extension of some word in $\Gamma$, but no word of $\Gamma$ is an extension of another word in $\Gamma$. By $|\Gamma|$ we denote the cardinality of $\Gamma$. Note 
that from the definition of $\Gamma$ it follows that a finite maximal antichain does not contain the empty word $\emptyset$ as all words are extensions of $\emptyset$.

Let us now state and prove the following lemma.

\section{LEMMA 3.4}

Let $0<r<+\infty$ and $\kappa_{r}$ be as in Lemma 3.2. Then, for any finite maximal antichain $\Gamma$, we have

(a) $L^{-1} \sum_{\sigma \in \Gamma} \mu_{h}\left(J_{\sigma}\right) \mu_{h} \circ \varphi_{\sigma}^{-1} \leq \mu_{h} \leq L \sum_{\sigma \in \Gamma} \mu_{h}\left(J_{\sigma}\right) \mu_{h} \circ \varphi_{\sigma}^{-1}$, and

(b) $\left(L \xi^{r}\right)^{-3 \kappa_{r} /\left(r+\kappa_{r}\right)} \leq \sum_{\sigma \in \Gamma}\left(\mu_{h}\left(J_{\sigma}\right)\left\|\varphi_{\sigma}^{\prime}\right\|^{r}\right)^{\kappa_{r} /\left(r+\kappa_{r}\right)} \leq\left(L \xi^{r}\right)^{3 \kappa_{r} /\left(r+\kappa_{r}\right)}$.

\section{Proof}

(a) Let $M=\max \{|\sigma|: \sigma \in \Gamma\}$. Note that the Borel $\sigma$-algebra on $E$ is generated by the set $\left\{J_{\sigma}: \sigma \in \Omega\right\}$ of all basic intervals. For any two basic intervals, either they are disjoint or one is contained in the other. Hence, it is enough to prove that, for any $\omega \in \Omega_{n}$ with $n \geq M$,

$$
L^{-1} \sum_{\sigma \in \Gamma} \mu_{h}\left(J_{\sigma}\right) \mu_{h} \circ \varphi_{\sigma}^{-1}\left(J_{\omega}\right) \leq \mu_{h}\left(J_{\omega}\right) \leq L \sum_{\sigma \in \Gamma} \mu_{h}\left(J_{\sigma}\right) \mu_{h} \circ \varphi_{\sigma}^{-1}\left(J_{\omega}\right)
$$

which follows along similar lines as the proof of [R2, Lemma 3.4(a)].

(b) As $\Gamma$ is a finite maximal antichain, there exists a finite sequence of positive integers $n_{1}<n_{2}<\cdots<n_{K}$ such that

$$
\Gamma=\Gamma_{1} \cup \Gamma_{2} \cup \cdots \cup \Gamma_{K},
$$

where $\Gamma_{j}=\left\{\sigma \in \Gamma:|\sigma|=n_{j}\right\}$ for all $1 \leq j \leq K$. Let $M$ be a positive integer such that $M \geq n_{K}$. Then by Lemma 3.3, we have

$$
\begin{aligned}
& \sum_{\sigma \in \Gamma}\left(\mu_{h}\left(J_{\sigma}\right)\left\|\varphi_{\sigma}^{\prime}\right\|^{r}\right)^{\kappa_{r} /\left(r+\kappa_{r}\right)} \\
& \geq \sum_{j=1}^{K} \sum_{\tau \in \Gamma_{j}}\left(\mu_{h}\left(J_{\tau}\right)\left\|\varphi_{\tau}^{\prime}\right\|^{r}\right)^{\kappa_{r} /\left(r+\kappa_{r}\right)}\left(L \xi^{r}\right)^{-\kappa_{r} /\left(r+\kappa_{r}\right)} \\
& \times \sum_{\sigma \in \Omega_{M-n_{j}}}\left(\mu_{h}\left(J_{\sigma}\right)\left\|\varphi_{\sigma}^{\prime}\right\|^{r}\right)^{\kappa_{r} /\left(r+\kappa_{r}\right)} \\
& \geq\left(L \xi^{r}\right)^{-\kappa_{r} /\left(r+\kappa_{r}\right)} \sum_{j=1}^{K} \sum_{\tau \in \Gamma_{j}}\left(\mu_{h}\left(J_{\tau}\right)\left\|\varphi_{\tau}^{\prime}\right\|^{r}\right)^{\kappa_{r} /\left(r+\kappa_{r}\right)} \\
& \times \sum_{\sigma \in \Omega_{M-n_{j}}}\left(\mu_{h}\left(J_{\sigma}\right)\left\|\varphi_{\sigma}^{\prime}\right\|^{r}\right)^{\kappa_{r} /\left(r+\kappa_{r}\right)} \\
&=\left(L \xi^{r}\right)^{-\kappa_{r} /\left(r+\kappa_{r}\right)} \sum_{j=1}^{K} \sum_{\tau \in \Gamma_{j}} \sum_{\sigma \in \Omega_{M-n_{j}}}\left(\mu_{h}\left(J_{\tau}\right)\left\|\varphi_{\tau}^{\prime}\right\|^{r}\right)^{\kappa_{r} /\left(r+\kappa_{r}\right)}
\end{aligned}
$$




$$
\begin{aligned}
& \times\left(\mu_{h}\left(J_{\sigma}\right)\left\|\varphi_{\sigma}^{\prime}\right\|^{r}\right)^{\kappa_{r} /\left(r+\kappa_{r}\right)} \\
\geq & \left(L \xi^{r}\right)^{-2 \kappa_{r} /\left(r+\kappa_{r}\right)} \sum_{j=1}^{K} \sum_{\tau \in \Gamma_{j}} \sum_{\sigma \in \Omega_{M-n_{j}}}\left(\mu_{h}\left(J_{\tau \sigma}\right)\left\|\varphi_{\tau \sigma}^{\prime}\right\|^{r}\right)^{\kappa_{r} /\left(r+\kappa_{r}\right)} \\
= & \left(L \xi^{r}\right)^{-2 \kappa_{r} /\left(r+\kappa_{r}\right)} \sum_{\sigma \in \Omega_{M}}\left(\mu_{h}\left(J_{\sigma}\right)\left\|\varphi_{\sigma}^{\prime}\right\|^{r}\right)^{\kappa_{r} /\left(r+\kappa_{r}\right)} \\
\geq & \left(L \xi^{r}\right)^{-3 \kappa_{r} /\left(r+\kappa_{r}\right)} .
\end{aligned}
$$

Similarly, we have $\sum_{\sigma \in \Gamma}\left(\mu_{h}\left(J_{\sigma}\right)\left\|\varphi_{\sigma}^{\prime}\right\|^{r}\right)^{\kappa_{r} /\left(r+\kappa_{r}\right)} \leq\left(L \xi^{r}\right)^{3 \kappa_{r} /\left(r+\kappa_{r}\right)}$. Hence, the lemma is obtained.

Let us now give the following lemma.

\section{LEMMA 3.5}

Let $x, y \in J$, and let $\sigma \in \Omega$. Then

$$
\xi^{-1}\left\|\varphi_{\sigma}^{\prime}\right\| d(x, y) \leq d\left(\varphi_{\sigma}(x), \varphi_{\sigma}(y)\right) \leq\left\|\varphi_{\sigma}^{\prime}\right\| d(x, y)
$$

where $\xi$ is the constant of Lemma 2.2.

\section{Proof}

By the mean value theorem, for any $x, y \in J$ there exists some $w \in(x, y)$ such that

$$
d\left(\varphi_{\sigma}(x), \varphi_{\sigma}(y)\right)=\left|\varphi_{\sigma}^{\prime}(w)\right| d(x, y),
$$

and so, by Proposition 2.4, for any $z \in J$,

$$
\xi^{-1}\left|\varphi_{\sigma}^{\prime}(z)\right| d(x, y) \leq d\left(\varphi_{\sigma}(x), \varphi_{\sigma}(y)\right)=\left|\varphi_{\sigma}^{\prime}(w)\right| d(x, y) \leq\left\|\varphi_{\sigma}^{\prime}\right\| d(x, y) .
$$

Now take the supremum over all $z \in J$, and then

$$
\xi^{-1}\left\|\varphi_{\sigma}^{\prime}\right\| d(x, y) \leq d\left(\varphi_{\sigma}(x), \varphi_{\sigma}(y)\right) \leq\left\|\varphi_{\sigma}^{\prime}\right\| d(x, y)
$$

to obtain the assertion of the lemma.

Using the above lemma and Lemma 3.4(a), and the parallel lines as [R2, Lemma 3.5], the following lemma can be proved.

\section{LEMMA 3.6}

Let $\Gamma \subset \Omega$ be a finite maximal antichain, let $n \in \mathbb{N}$ with $n \geq|\Gamma|$, and let $0<r<$ $+\infty$. Then $V_{n, r}\left(\mu_{h}\right) \leq \inf \left\{L \sum_{\sigma \in \Gamma} \mu_{h}\left(J_{\sigma}\right)\left\|\varphi_{\sigma}^{\prime}\right\|^{r} V_{n_{\sigma}, r}\left(\mu_{h}\right): 1 \leq n_{\sigma}, \sum_{\sigma \in \Gamma} n_{\sigma} \leq\right.$ $n\}$.

By using Lemma 3.6 and proceeding along lines similar to [R2, Proposition 3.6], the following proposition can be proved. 
PROPOSITION 3.7

Let $0<r<+\infty$ and $\kappa_{r}$ be as in Lemma 3.2. Then $\lim \sup _{n} n V_{n, r}^{\kappa_{r} / r}\left(\mu_{h}\right)<+\infty$.

\section{NOTE 3.8}

We say that the cookie-cutter mapping $f$ satisfies the $O S C$ if the corresponding set of branch inverses $\left\{\varphi_{1}, \varphi_{2}, \ldots, \varphi_{N}\right\}$ satisfies the OSC: there exists a bounded nonempty open set $U \subset J$ (in the topology of $J$ ) such that $\varphi_{j}(U) \subset U$ and $\varphi_{i}(U) \cap$ $\varphi_{j}(U)=\emptyset$ for $1 \leq i \neq j \leq N$. Furthermore, $f$ satisfies the strong OSC (SOSC) if $U$ can be chosen such that $U \cap E \neq \emptyset$. Note that we can choose $U=(0,1)$, and so, because of the net properties of the basic intervals of the cookie-cutter set, it follows that the cookie-cutter mapping $f$ satisfies the SOSC.

As a parallel to [R2, Lemma 3.7] the following lemma can be proved.

LEMMA 3.9

Let $\Gamma \subset \Omega$ be a finite maximal antichain. Then there exists $n_{0}=n_{0}(\Gamma)$ such that for every $n \geq n_{0}$ there exists a set of positive integers $\left\{n_{\sigma}:=n_{\sigma}(n)\right\}_{\sigma \in \Gamma}$ such that $\sum_{\sigma \in \Gamma} n_{\sigma} \leq n$ and

$$
u_{n, r}\left(\mu_{h}\right) \geq\left(L \xi^{r}\right)^{-1} \sum_{\sigma \in \Gamma} \mu_{h}\left(J_{\sigma}\right)\left\|\varphi_{\sigma}^{\prime}\right\|^{r} u_{n_{\sigma}, r}\left(\mu_{h}\right) .
$$

Let us now prove the following proposition, which shows that the $\kappa_{r}$-dimensional lower quantization coefficient of order $r$ of the probability measure $\mu_{h}$ is positive.

PROPOSITION 3.10

Let $\mu_{h}$ be the Gibbs-like measure, and let $0<r<+\infty$. Moreover, let $\kappa_{r}$ be as in Lemma 3.2. Then $\liminf _{n} n V_{n, r}^{\kappa_{r} / r}\left(\mu_{h}\right)>0$.

Proof

Let $\Gamma$ be a finite maximal antichain. By Lemma 3.9, we have $n_{0}$ and for $n \geq n_{0}$ the numbers $\left\{n_{\sigma}:=n_{\sigma}(n)\right\}_{\sigma \in \Gamma}$ which satisfy the conclusion of the lemma. Set $c=\min \left\{n^{r / \kappa_{r}} u_{n, r}\left(\mu_{h}\right): n \leq n_{0}\right\}$. Clearly each $u_{n, r}\left(\mu_{h}\right)>0$, and hence $c>0$. Suppose that $n \geq n_{0}$, and suppose that $k^{r / \kappa_{r}} u_{k, r}\left(\mu_{h}\right) \geq c$ for all $k<n$. Hence, using Lemma 3.9, we have

$$
\begin{aligned}
& n^{r / \kappa_{r}} u_{n, r}\left(\mu_{h}\right) \\
& \quad \geq n^{r / \kappa_{r}}\left(L \xi^{r}\right)^{-1} \sum_{\sigma \in \Gamma} \mu_{h}\left(J_{\sigma}\right)\left\|\varphi_{\sigma}^{\prime}\right\|^{r} u_{n_{\sigma}, r}\left(\mu_{h}\right) \\
& \quad=n^{r / \kappa_{r}}\left(L \xi^{r}\right)^{-1} \sum_{\sigma \in \Gamma} \mu_{h}\left(J_{\sigma}\right)\left\|\varphi_{\sigma}^{\prime}\right\|^{r}\left(n_{\sigma}(n)\right)^{-r / \kappa_{r}}\left(n_{\sigma}(n)\right)^{r / \kappa_{r}} u_{n_{\sigma}, r}\left(\mu_{h}\right) \\
& \quad \geq c\left(L \xi^{r}\right)^{-1} \sum_{\sigma \in \Gamma} \mu_{h}\left(J_{\sigma}\right)\left\|\varphi_{\sigma}^{\prime}\right\|^{r}\left(\frac{n_{\sigma}(n)}{n}\right)^{-r / \kappa_{r}} .
\end{aligned}
$$


Using Hölder's inequality (with exponents less than 1), we have

$$
\begin{aligned}
n^{r / \kappa_{r}} u_{n, r}\left(\mu_{h}\right) \geq & c\left(L \xi^{r}\right)^{-1}\left(\sum_{\sigma \in \Gamma}\left(\mu_{h}\left(J_{\sigma}\right)\left\|\varphi_{\sigma}^{\prime}\right\|^{r}\right)^{\kappa_{r} /\left(r+\kappa_{r}\right)}\right)^{\left(1+r / \kappa_{r}\right)} \\
& \times\left(\sum_{\sigma \in \Gamma}\left(\frac{n_{\sigma}(n)}{n}\right)^{\left(-r / \kappa_{r}\right)\left(-\kappa_{r} / r\right)}\right)^{-r / \kappa_{r}} \cdot
\end{aligned}
$$

By Lemma 3.4(b) and the fact that $\sum_{\sigma \in \Gamma} n_{\sigma}(n) \leq n$, we have

$$
n^{r / \kappa_{r}} u_{n, r}\left(\mu_{h}\right) \geq c\left(L \xi^{r}\right)^{-1}\left(L \xi^{r}\right)^{-3} .
$$

Therefore, by induction,

$\liminf _{n} n u_{n, r}^{\kappa_{r} / r}\left(\mu_{h}\right) \geq\left[c\left(L \xi^{r}\right)^{-4}\right]^{\kappa_{r} / r}>0 ;$ that is, $\liminf _{n} n V_{n, r}^{\kappa_{r} / r}\left(\mu_{h}\right)>0$,

and thus the proposition is obtained.

Proof of Theorem 3.1

Note that $e_{n, r}=V_{n, r}^{1 / r}$, and by [GL1, Proposition 11.3], we know the following:

(a) If $0 \leq t<\underline{D}_{r}<s$, then

$$
\lim _{n \rightarrow \infty} n e_{n, r}^{t}=+\infty \quad \text { and } \quad \liminf _{n \rightarrow \infty} n e_{n, r}^{s}=0 .
$$

(b) If $0 \leq t<\bar{D}_{r}<s$, then

$$
\limsup _{n \rightarrow \infty} n e_{n, r}^{t}=+\infty \quad \text { and } \quad \lim _{n \rightarrow \infty} n e_{n, r}^{s}=0 .
$$

From (a) and Proposition 3.10, we have $\kappa_{r} \leq \underline{D}_{r}$. From (b) and Proposition 3.7, we have $\bar{D}_{r} \leq \kappa_{r}$. Hence, $\kappa_{r} \leq \underline{D}_{r} \leq \bar{D}_{r} \leq \kappa_{r}$; that is, the quantization dimension $D_{r}$ exists and $D_{r}=\kappa_{r}$. Note that if $q_{r}=\kappa_{r} /\left(r+\kappa_{r}\right)$, by Lemma 3.2, we have $\beta\left(q_{r}\right)=r q_{r}$, and then $D_{r}=\beta\left(q_{r}\right) /\left(1-q_{r}\right)$. Moreover, by Proposition 3.10, we have $\liminf _{n} n V_{n, r}^{\kappa_{r} / r}\left(\mu_{h}\right)>0$. Thus, the proof of the theorem is complete.

\section{References}

[BW] J. A. Bucklew and G. L. Wise, Multidimensional asymptotic quantization with rth power distortion measures, IEEE Trans. Inform. Theory 28 (1982), 239-247. MR 0651819. DOI 10.1109/TIT.1982.1056486.

[F1] K. J. Falconer, The multifractal spectrum of statistically self-similar measures, J. Theoret. Probab. 7 (1994), 681-701. MR 1284660. DOI 10.1007/BF02213576.

[F2] Techniques in Fractal Geometry, Wiley, Chichester, UK, 1997. MR 1449135.

[GG] A. Gersho and R. M. Gray, Vector Quantization and Signal Compression, Kluwer, Boston, 1992. 
[GL1] S. Graf and H. Luschgy, Foundations of Quantization for Probability Distributions, Lecture Notes in Math. 1730, Springer, Berlin, 2000. MR 1764176. DOI 10.1007/BFb0103945.

[GL2] Asymptotics of the quantization errors for self-similar probabilities, Real Anal. Exchange 26 (2000/01), 795-810. MR 1844394.

[GL3] The quantization dimension of self-similar probabilities, Math. Nachr. 241 (2002), 103-109. MR 1912380. DOI 10.1002/1522-2616(200207)241:1<103::AID-MANA103〉3.0.CO;2-J.

[GN] R. M. Gray and D. L. Neuhoff, Quantization, IEEE Trans. Inform. Theory 44 (1998), 2325-2383. MR 1658787. DOI 10.1109/18.720541.

[HJK+] T. C. Halsey, M. H. Jensen, L. P. Kadanoff, I. Procaccia, and B. I. Shraiman, Fractal measures and their singularities: The characterization of strange sets, Phys. Rev. A (3) 33 (1986), 1141-1151. MR 0823474. DOI 10.1103/PhysRevA.33.1141.

[H] J. E. Hutchinson, Fractals and self-similarity, Indiana Univ. Math. J. 30 (1981), 713-747. MR 0625600. DOI 10.1512/iumj.1981.30.30055.

[LM] L. J. Lindsay and R. D. Mauldin, Quantization dimension for conformal iterated function systems, Nonlinearity 15 (2002), 189-199. MR 1877974. DOI 10.1088/0951-7715/15/1/309.

[P] N. Patzschke, Self-conformal multifractal measures, Adv. in Appl. Math. 19 (1997), 486-513. MR 1479016. DOI 10.1006/aama.1997.0557.

[R1] M. K. Roychowdhury, Quantization dimension for some Moran measures, Proc. Amer. Math. Soc. 138 (2010), 4045-4057. MR 2679625. DOI 10.1090/S0002-9939-2010-10406-9.

[R2] Quantization dimension function and Gibbs measure associated with Moran set, J. Math. Anal. Appl. 373 (2011), 73-82. MR 2684458. DOI 10.1016/j.jmaa.2010.06.021.

[WD] X. Wang and M. Dai, Mixed quantization dimension function and temperature function for conformal measures, Int. J. Nonlinear Sci. 10 (2010), 24-31. MR 2721065.

[Y] K. Yoshida, Functional Analysis, Springer, Berlin, 1966.

[Z] P. L. Zador, Development and evaluation of procedures for quantizing multivariate distributions, Ph.D. dissertation, Stanford University, Stanford, Calif., 1964. MR 2614227.

Department of Mathematics, University of Texas-Pan American, 1201 West University Drive, Edinburg, Texas 78539-2999, USA; roychowdhurymk@utpa.edu 